\title{
Identification of target gene of venous thromboembolism in patients with lymphoma via microarray analysis
}

\author{
PENGFEI LIU ${ }^{1}$, WENHUA JIANG ${ }^{2}$ and HUILAI ZHANG ${ }^{1}$ \\ ${ }^{1}$ Department of Lymphoma, Sino-US Center of Lymphoma and Leukemia, Tianjin Medical University Cancer Institute \\ and Hospital, National Clinical Research Center for Cancer, Key Laboratory of Cancer Prevention and Therapy, \\ Tianjin 300060; ${ }^{2}$ Department of Radiotherapy, Second Hospital of Tianjin Medical University, Tianjin 300211, P.R. China
}

Received February 17, 2016; Accepted January 6, 2017

DOI: $10.3892 / 01.2017 .6625$

\begin{abstract}
Patients with lymphoma are at high risk of developing venous thromboembolism (VTE). The purpose of the present study was to identify the target gene associated with VTE for patients with lymphoma. Microarray data was downloaded from the gene expression omnibus database (GSE17078), which comprised the control group, 27 normal blood outgrowth endothelial cell (BOEC) samples, and the case group, 3 BOEC samples of venous thrombosis with protein $\mathrm{C}$ deficiency. Differentially expressed genes (DEGs) were identified by the Limma package of R. Gene ontology (GO) and Kyoto encyclopedia of genes and genomes (KEGG) pathway analyses were performed via the database for annotation, visualization and integrated discovery. Differentially coexpressed pairs were identified by the DCGL package of R. The subsequent protein-protein interaction (PPI) networks and gene coexpression networks were constructed by the Search Tool for the Retrieval of Interacting Genes/Proteins database, and were visualized by Cytoscape software. A total of 110 DEGs were obtained, including 73 upregulated and 37 downregulated genes. GO and KEGG pathway enrichment analyses identified 132 significant GO terms and 9 significant KEGG pathways. In total, 97 PPI pairs for PPI network and 309 differential coexpression pairs for the gene coexpression network were obtained. Additionally, the connective tissue growth factor $(C T G F)$ gene was closely connected with other genes in the two networks. A total of 2 KEGG pathways were associated with VTE and CTGF may be the target gene of VTE in patients with lymphoma. The present study may identify the
\end{abstract}

Correspondence to: Dr Huilai Zhang, Department of Lymphoma, Sino-US Center of Lymphoma and Leukemia, Tianjin Medical University Cancer Institute and Hospital, National Clinical Research Center for Cancer, Key Laboratory of Cancer Prevention and Therapy, 24 Huan-Hu-Xi Road, Ti-Yuan-Bei, He Xi, Tianjin 300060, P.R. China

E-mail: snowlpf@163.com

Key words: venous thromboembolism, lymphoma, microarray, protein-protein interaction network, gene coexpression network molecular mechanism of VTE, but additional clinical study is required to validate the results.

\section{Introduction}

The high risk of venous thromboembolism (VTE) occurring in patients with cancer is a worldwide public health problem, particularly in patients with lymphoma, and leads to high mortality (1,2). Patients with cancer exhibit an 8-fold higher risk of mortality compared with patients without cancer in incidences of acute VTE (3), and patients with carcinogenic malignancies were more prone to developing VTE (4). Additionally, compared to patients with other types of cancer, patients with lymphoma have been reported exhibit a higher frequency of VTE (7.7\%) (5). The clinical risk factors for VTE comprise exogenous and endogenous factors: Exogenous factors include surgery and chemotherapy, immobility, trauma and hormone use, and endogenous factors include cancer, inherited elevated risk (family history of VTE) and hypercoagulation (6).

Lymphoma are a heterogeneous group of lymphoid disorders that originate in the lymphatic tissues and share a common characteristic of clonal expansion of malignant lymphocytes (7). Although numerous types of cancer eventually spread to parts of the lymphatic system, lymphoma is different as it actually originates in this area $(8,9)$. A previous study demonstrated that the greatest risk of VTE occurred in patients with lymphoma, followed by patients with lung and gastrointestinal cancer, which suggests that the incidence of VTE in patients with lymphoma was higher compared with patients with solid tumors (10). The majority of patients with lymphoma receive anti-neoplastic therapy and thus increase their risk of VTE (2). A previous study suggested that the rate of VTE was $59.5 \%$ in patients with lymphoma, and that it occurred earlier due to the period of intensive therapy compared with patients with VTE without lymphoma (11). However, there are few studies examining procoagulants and the molecular mechanism of VTE in patients with malignant lymphoma. The risk factors of VTE in lymphoma patients have not been fully characterized, and the majority of thromboses occur early in the course of the disease (12).

The use of anticoagulation may lead to an increased incidence of bleeding, which may result in thromboprophylaxis. 
Analysis of the risk factors of thrombosis may allow appropriate prophylaxis of VTE occurrence, to protect against the fatal or detrimental consequences, such as bleeding or mortality (12). Although some risk factors such as the site of cancer, platelet count, level of hemoglobin, leukocyte count and body mass index have been proposed, genetic risk factors should also be investigated (13). To date, a small number of studies have evaluated congenital thrombophilia in patients with lymphoma $(14,15)$. A previous study found that there was an increased risk of venous thrombosis in patients with cancer and demonstrated that patients possessing the Factor V Leiden mutation exhibited a 12-fold increase of thrombosis, whilst patients without this mutation only had 5-fold increase (10).

However, the molecular pathogenesis of thrombosis in patients with lymphoma remains unknown. In the present study, the gene associated with VTE in patients with lymphoma was identified by bioinformatic approaches, which may provide novel insights for additional application of thromboprophylaxis.

\section{Materials and methods}

Microarray data preprocessing. Microarray data (GSE17078) was downloaded from the Gene Expression Omnibus (GEO) database (http://www.ncbi.nlm.nih.gov/geo/) and contained 27 normal blood outgrowth endothelial cell (BOECs) samples, the control group and 3 BOECs samples of venous thrombosis with Protein $\mathrm{C}$ deficiency, the case group. The affy package of $\mathrm{R}$ was used to normalize the raw data. Probes were switched into gene symbols with microarray platform of GPL96 [HG-U133A] Affymetrix Human Genome U133A Array. Probes without gene symbols and expression values were filtered.

Identification of differentially expressed genes and functional enrichment analysis. With the cut-offs of adjusted to $\mathrm{P}<0.05$ and an absolute value of log (fold change) of $>1$, differentially expressed genes (DEGs) were identified by the limma package of R. The following gene ontology (GO) and Kyoto encyclopedia of genes and genomes (KEGG) pathway analyses were performed by the Database for Annotation, Visualization and Integrated Discovery (DAVID; david.abcc.ncifcrf.gov) for annotation, visualization and integrated discovery. GO described DEGs in terms of their associated biological processes, cellular components and molecular functions in a species-independent manner. The KEGG database is used to display the biochemical pathways of the DEGs of interest.

Identification of differential coexpression pairs. DCGL is a package that may serve as an effective tool for differential coexpression analysis (DCEA). DCEA determines the change of expression correlation of gene pairs and aids the investigation of the global transcriptional mechanisms underlying phenotypic changes (16). In the present study, differential coexpression pairs were identified by the DCGL package (version 2.1.2) (16) of $\mathrm{R}$ and $\mathrm{P}<0.05$ was used as the cut-off criterion.

Construction of protein-protein interaction (PPI) network and gene coexpression network. Therefore, PPI and gene coexpression networks were constructed by the Search Tool for the Retrieval of Interacting Genes/Proteins (STRING) database and visualized by Cytoscape (version 3.4.0).

\section{Results}

DEGs. A total of 110 DEGs were obtained, including 73 upregulated and 37 downregulated genes. A volcano plot of DEGs is demonstrated in Fig. 1. Genes were arranged along axes of biological and statistical significance in the volcano plot.

Functional enrichment analysis. GO enrichment analyses illustrated that the DEGs were enriched in 132 GO terms. A total of $98 \mathrm{GO}$ terms were in biological processes, 18 terms in molecular function and 16 terms in cellular component. Additionally, 9 significant KEGG pathways were identified, including the two most significant types of pathways: The intestinal immune network for IgA production and the cytokine-cytokine receptor interaction pathway. The nine KEGG pathways are listed in Table I.

Differential coexpression pairs. A total of 309 differential coexpression pairs were identified by the DCGL package, which included 166 positive correlative regulation of coexpression pairs, 80 upregulated and 86 downregulated simultaneously, and 143 negative correlative regulation of coexpression pairs.

PPI network and gene coexpression network. The PPI and coexpression networks were constructed by STRING, as demonstrated in Fig. 2. The degree of the node was calculated based on the number of links of 1 node, which directly contacted with other nodes. The $\mathrm{C}-\mathrm{x}-\mathrm{C}$ motif chemokine ligand 12 (CXCL12), amyloid $\beta$ precursor protein (APP), Vvon Willebrand factor $(V W F)$, apolipoprotein $\mathrm{E}(A P O E)$, bone morphogenic protein 4 (BMP4), thrombospondin 1 (THBS1), peroxisome proliferator activated receptor $\gamma(P P A R G)$ and connective tissue growth factor genes $(C T G F)$ exhibited high degrees in PPI network. The coexpression network contained 309 coexpression pairs and 105 genes (Fig. 3). The lymphocyte cytosolic protein 1 ( $L C P 1)$, popeye domain containing 3 (POPDC3), nuclear receptor subfamily 2 group F member 2 (NR2F2), Yip1 domain family member 5 (YIPF5), epiregulin $(E R E C)$, glutaminyl-peptide cyclotransferase ( $Q P C T), \mathrm{nBAF}$ chromatin remodeling complex subunit (SS18), neuron navigator 2 (NAV2), podoplanin $(P D P N), \mathrm{CTGF}$, nuclear factor I B $(N F I B)$ and perilipin 3 genes (PLIN3) possessed high degrees $>10$ in this coexpression network.

\section{Discussion}

The occurrence of VTE associated with cancer is a common complication of patients with malignancies (17). The clinical relevance of the VTE phenomenon is associated with the presence of cancer itself and solid tumors are typically considered to present higher risks (18-20). However, a number of previous studies have suggested that the high risk of VTE associated with patients with hematological malignancies such as lymphoma and leukemia may be comparable to patients with solid tumors $(10,21)$. In fact, the incidence of VTE in 
Table I. Significant KEGG pathways.

\begin{tabular}{|c|c|c|c|c|}
\hline Category & Pathway Name & $\begin{array}{l}\text { Gene } \\
\text { Number }\end{array}$ & P-value & Genes \\
\hline KEGG_PATHWAY & $\begin{array}{l}\text { Intestinal immune network } \\
\text { for Immunoglobulin } \\
\text { A production }\end{array}$ & 5 & 0.001001303 & $\begin{array}{l}\text { HLA-DPA1, } \\
\text { HLA-DPB1, ITGA4, } \\
\text { HLA-DMA, CXCL12 }\end{array}$ \\
\hline KEGG_PATHWAY & $\begin{array}{l}\text { Cytokine-cytokine } \\
\text { receptor interaction }\end{array}$ & 8 & 0.009523061 & $\begin{array}{l}\text { IL1R1, CXCL5, CCL20, } \\
\text { IL10RB, CXCL3, KITLG, } \\
\text { CXCL6, CXCL12 }\end{array}$ \\
\hline KEGG_PATHWAY & Viral myocarditis & 4 & 0.027292882 & $\begin{array}{l}\text { RAC2, HLA-DPA1, } \\
\text { HLA-DPB1, HLA-DMA }\end{array}$ \\
\hline KEGG_PATHWAY & $\begin{array}{l}\text { Chemokine } \\
\text { signaling pathway }\end{array}$ & 6 & 0.028009403 & $\begin{array}{l}\text { RAC2, CXCL5, CCL20, } \\
\text { CXCL3, CXCL6, CXCL12 }\end{array}$ \\
\hline KEGG_PATHWAY & Asthma & 3 & 0.028974901 & $\begin{array}{l}\text { HLA-DPA1, HLA-DPB1, } \\
\text { HLA-DMA }\end{array}$ \\
\hline KEGG_PATHWAY & Cell adhesion molecules & 5 & 0.032728891 & $\begin{array}{l}\text { HLA-DPA1, HLA-DPB1, } \\
\text { ITGA4, SELE, HLA-DMA }\end{array}$ \\
\hline KEGG_PATHWAY & $\begin{array}{l}\text { Antigen processing } \\
\text { and presentation }\end{array}$ & 4 & 0.040649303 & $\begin{array}{l}\text { PDIA3, HLA-DPA1, } \\
\text { HLA-DPB1, HLA-DMA }\end{array}$ \\
\hline KEGG_PATHWAY & Allograft rejection & 3 & 0.043169157 & $\begin{array}{l}\text { HLA-DPA1, HLA- } \\
\text { DPB1, HLA-DMA }\end{array}$ \\
\hline KEGG_PATHWAY & Graft-versus-host disease & 3 & 0.049900633 & $\begin{array}{l}\text { HLA-DPA1, HLA-DPB1, } \\
\text { HLA-DMA }\end{array}$ \\
\hline
\end{tabular}

KEGG, Kyoto Encyclopedia of Genes and Genomes.

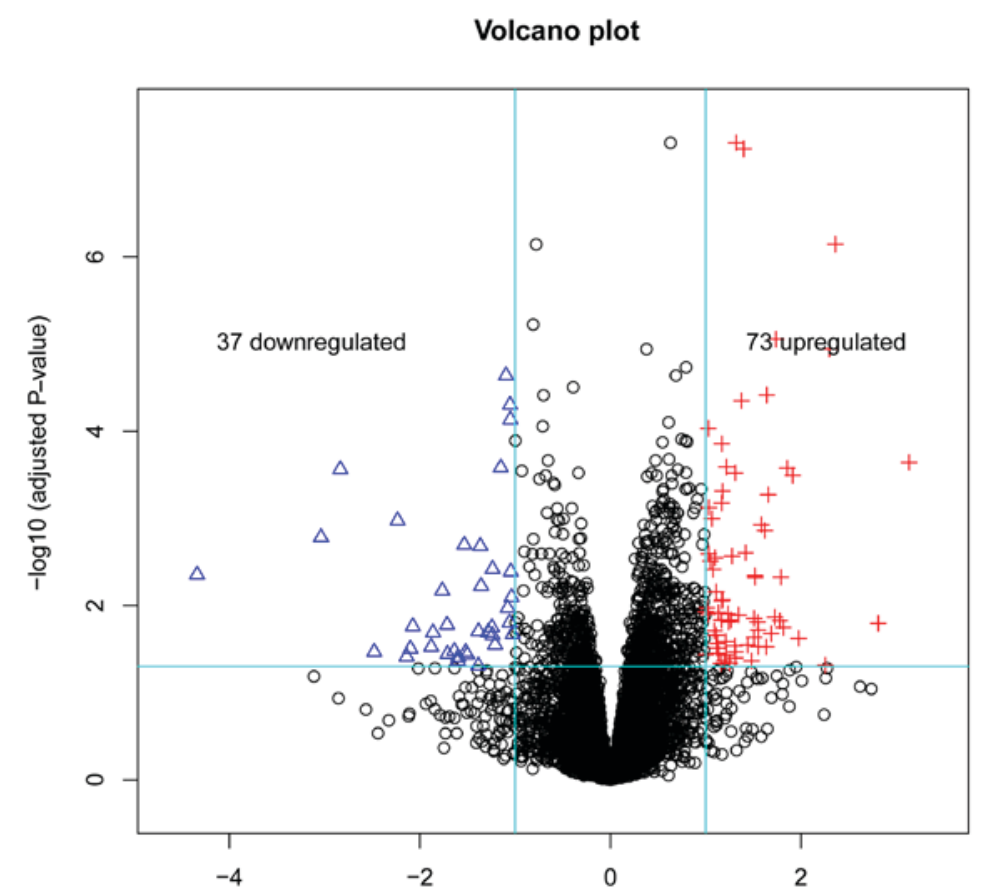

Figure 1. Volcano plot of DEGs. The horizontal axis was the fold change between case and control groups, and exhibits the change in biological impact, whereas the vertical axis represents the reliability of the adjusted P-value. Black circles represent non-differentially expressed genes, blue triangles are downregulated genes and red + signs are upregulated genes.

hematological-associated malignancies exceeded the incidence rate of solid tumors $(22,23)$. A previous study revealed that the global incidence rate of thrombosis in patients with lymphoma was $>6 \%$, and the majority of thromboses occurred during the 
3316

LIU et al: TARGETED GENE OF VENOUS THROMBOEMBOLISM IN PATIENTS WITH LYMPHOMA

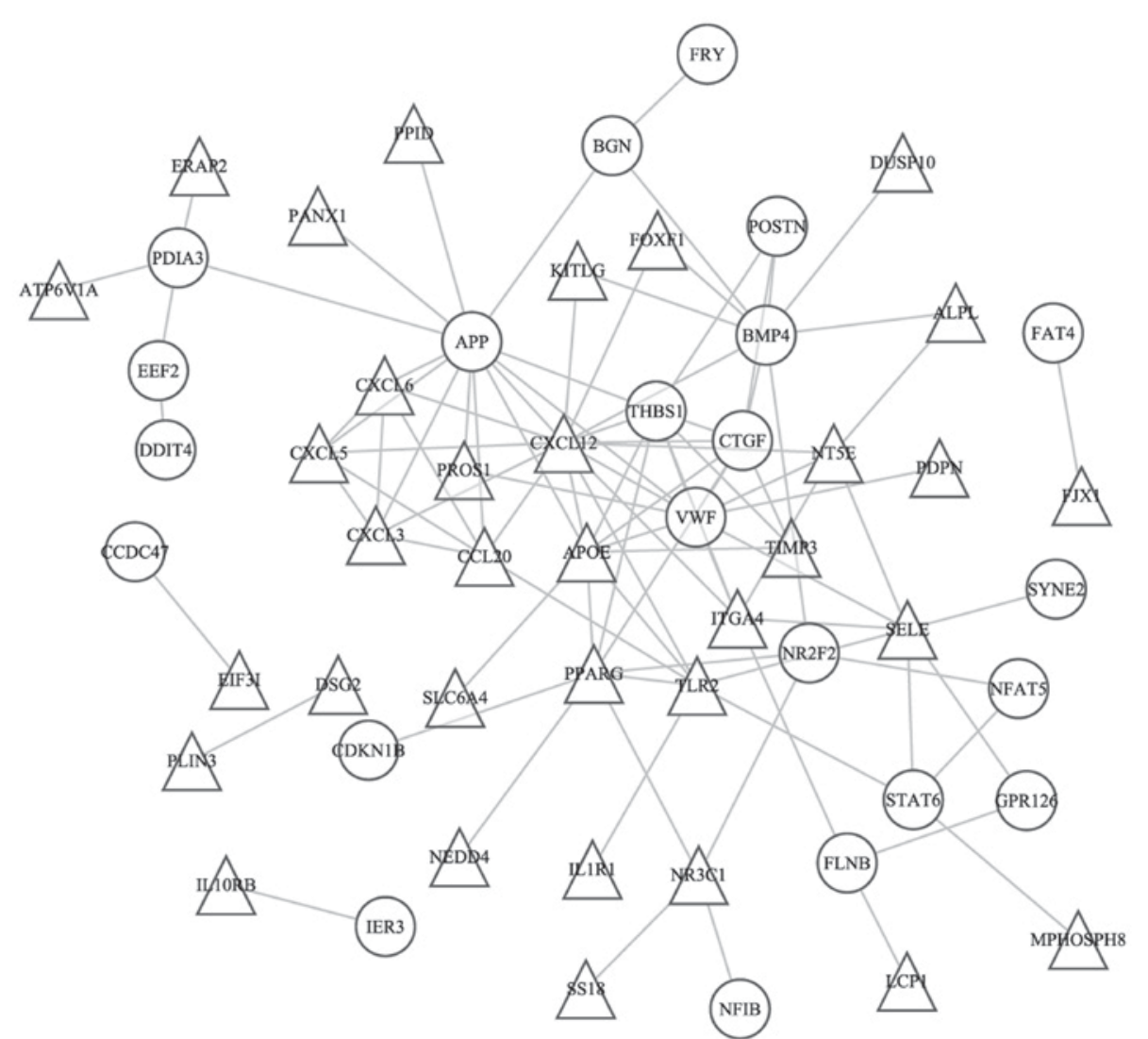

Figure 2. Protein -protein interaction network of venous thromboembo
for downregulated genes and 22 triangle nodes for unregulated genes.

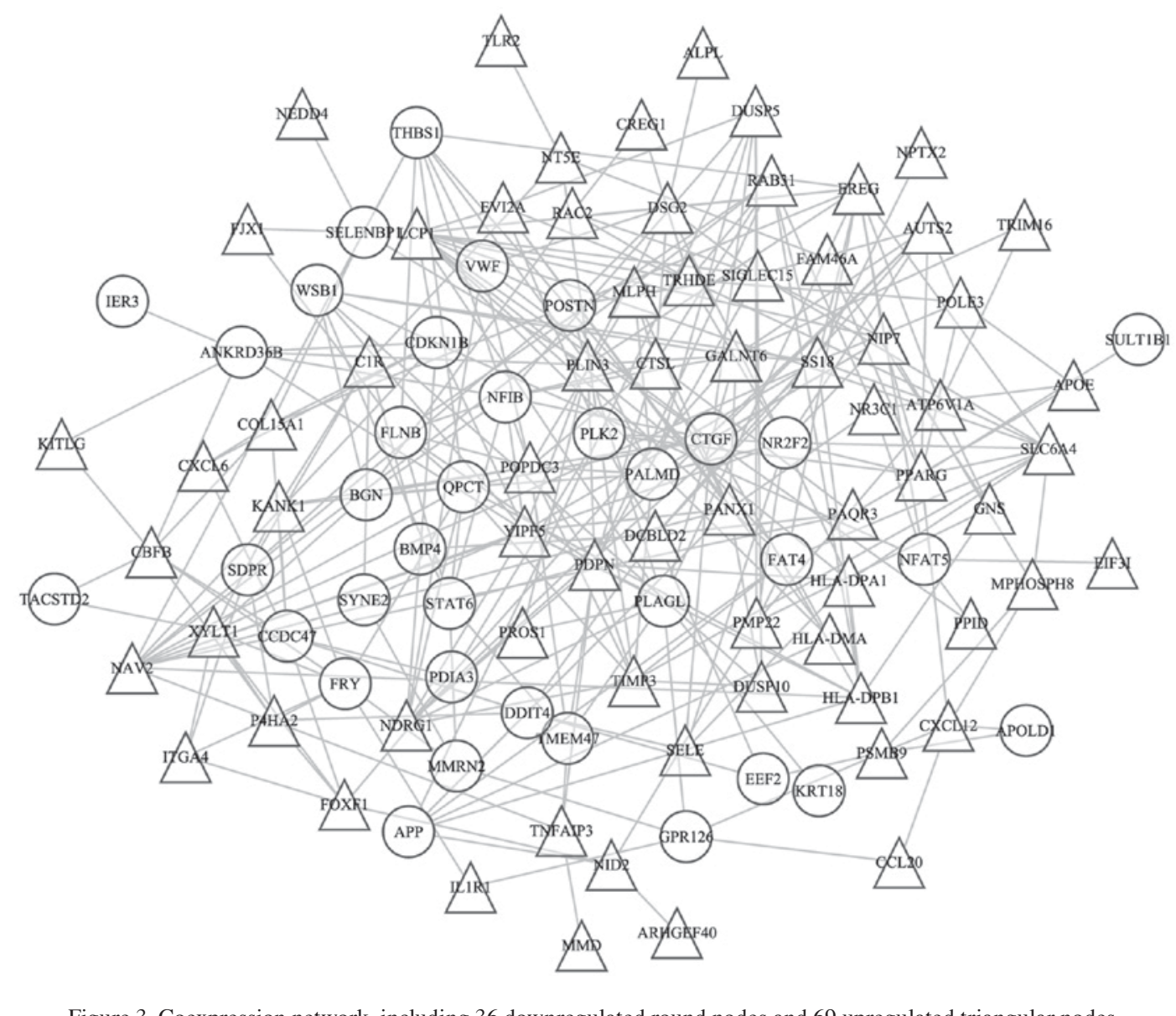


treatment stages of this disease (12). Therefore, microarray data was analyzed by bioinformatics to investigate the molecular mechanism of VTE in patients with lymphoma, which may provide novel insights for antithrombotic prophylaxis.

In total, two significant KEGG pathways, the intestinal immune network for IgA production and the cytokine-cytokine receptor interaction pathway, were identified in the present study. The intestines are the largest lymphoid tissue in the body, and serve a critical role in intestinal immunity: A number of non-inflammatory immunoglobulin A (IgA) antibodies are produced by the immunological factors in the intestines as the first line of defense against foreign microorganisms (24). Secreted IgA molecules may promote immune exclusion via entrapping dietary antigens and microorganisms in the mucus, and function to neutralize pathogenic microbes and toxins (25). Cytokines, which are soluble extracellular proteins or glycoproteins, are crucial intercellular regulators and mobilizers of cells that are involved in the innate and adaptive immune responses, cell death, cell growth, differentiation, angiogenesis and the repair processes involved in the restoration of homeostasis (26). However, the underlying mechanisms of complex diseases such as lymphoma and VTE are affected by a number of environmental and genetic factors (27). Therefore, the PPI and gene coexpression networks were utilized to illustrate the molecular basis of lymphoma. It was notable that the PPI network was used to identify the differences between normal and disease patient groups and helped to recognize potentially disease-associated gene candidates (28). The current computational approach to predicting PPI networks was fast and useful for selecting potential targets for additional experimental screening (29). The gene coexpression network may provide potential specific molecular mechanisms in disease. It is common to use gene coexpression networks to recognize highly connected genes, which are usually associated with diseases (30).

PPI networks usually serve an important role in identifying novel protein functions (31) and examining the associations between protein network structure and function (32). Gene expression profiles may describe the pairwise associations of proteins with a biological network. Similarly, gene coexpression networks may be used to describe genes that exhibit similar expression patterns in regulatory processes, pathways and certain complexes (33). Gene coexpression network is based on a gene coexpression measure and used for gene filtering and outcome prediction. The main feature of these two networks is the degree distribution- the number of connections a node possesses. Key nodes, such as hubs, which possess high degrees are highly connected with other genes and may be potential disease targets (34). The results of the present study suggest that $C T G F$ possessed the highest degree in the PPI and coexpression networks. Therefore, it was logical to hypothesize that $C T G F$ was the target gene of VTE. $C T G F$, also termed CCN2, is a member of the $\mathrm{CCN}$ family (35). CTGF has been demonstrated to serve important roles in a number of biological processes such as cell proliferation, differentiation, adhesion, angiogenesis, tissue wound repair and fibrotic disease (36). It is a secreted multifunctional matricellular protein comprising four distinct conserved structural modules that include a thrombospondin type 1 repeat (TSR), a von Willebrand type $\mathrm{C}$ repeats domain, an amino-terminal insulin-like growth factor binding domain and a carboxyl-terminal (CT) cysteine-knot motif (37). These structural modules of $C T G F$ confer a variety of functions. For instance, the TSR domain interacts with vascular endothelial growth factor (38) and the CT domain binds to transforming growth factor (TGF)- $\beta$ superfamily (39). In addition, $C T G F$ has been identified in almost all fibrotic pathologies (40): It has been revealed that $C T G F$ is upregulated during the pathogenesis of several fibrotic diseases such as kidney and liver fibrosis, scleroderma and atherosclerosis (41). It was also indicated that $C T G F$ may induce sustained fibrosis during interaction with TGF- $\beta$ (42) and intensify the production of the extracellular matrix, which is associated with other fibrosis-inducing conditions (40). Additionally, fibrosis, cardiovascular diseases (41) and numerous types of malignancy (43) were associated with aberrant $C T G F$ expression. In pancreatic cancer, $C T G F$-specific antibodies diminished tumor growth and metastases (44). However, in hematological malignancies, overexpressed $C T G F$ was associated with poor outcome in patients with precursor-B acute lymphoblastic leukemia (45).

Evidence suggests that the tumor type, the stage of the cancer and treatment with antineoplastic agents may contribute to the absolute risk of VTE development (46). Furthermore, age, immobilization, surgery and other comorbidities will also affect the overall likelihood of thrombotic complications, and even affect patients without cancer. At present, the mechanisms of hereditary thrombophilia in patients with cancer and thrombosis remain unclear. Additional studies will assist to improve the prophylactic and treatment strategies for VTE in these complex patients.

In conclusion, the present study screened important genes, which may reveal the molecular mechanisms of VTE in patients with lymphoma. These results may help to define the lymphoma populations at high thrombotic risk, who require the development of effective prophylactic treatments. However, confirmation of these findings and additional studies investigating lymphoma are required.

\section{Acknowledgements}

The present study was supported by the Health Bureau Science and Technology Foundation of Tianjin (grant no., 2014KZ102) and the Municipal Science and Technology Commission of Tianjin (grant no. 15ZLZLZF00440 and 16ZLZXZF00120).

\section{References}

1. Khorana AA, Francis CW, Culakova E, Kuderer NM and Lyman GH: Frequency, risk factors, and trends for venous thromboembolism among hospitalized cancer patients. Cancer 110: 2339-2346, 2007.

2. Wun T and White RH: Venous thromboembolism in patients with acute leukemia, lymphoma and multiple myeloma. Thromb Res 125 (Suppl 2): S96-S102, 2010.

3. Zhou X, Teegala S, Huen A, Ji Y, Fayad L, Hagemeister FB, Gladish G and Vadhan-Raj S: Incidence and risk factors of venous thromboembolic events in lymphoma. Am J Med 123: 935-941, 2010.

4. Khalil J, Bensaid B, Elkacemi H, Afif M, Bensaid Y, Kebdani T and Benjaafar $\mathrm{N}$ : Venous thromboembolism in cancer patients: An underestimated major health problem. World J Surg Oncol 13: 204, 2015. 
5. Mohren M, Markmann I, Jentsch-Ullrich K, Koenigsmann M, Lutze $\mathrm{G}$ and Franke A: Increased risk of thromboembolism in patients with malignant lymphoma: A single-centre analysis. $\mathrm{Br}$ J Cancer 92: 1349-1351, 2005.

6. Cushman M: Epidemiology and risk factors for venous thrombosis. Semin Hematol 44: 62-69, 2007.

7. Zheng RL, Jiang YJ and Wang X: Role of microRNAs on therapy resistance in Non-Hodgkin's lymphoma. Int J Clin Exp Med 7: 3818-3832, 2014

8. Benson N, Whipple M and Kalet IJ: A Markov model approach to predicting regional tumor spread in the lymphatic system of the head and neck. AMIA Annu Symp Proc 2006: 31-35, 2006.

9. Kewitz S, Kurch L, Volkmer I and Staege MS: Stimulation of the hypoxia pathway modulates chemotherapy resistance in Hodgkin's lymphoma cells. Tumor Biol 37: 8229-8237, 2016.

10. Blom JW, Doggen CJ, Osanto S and Rosendaal FR: Malignancies, prothrombotic mutations, and the risk of venous thrombosis JAMA 293: 715-722, 2005.

11. Goldschmidt N, Linetsky E, Shalom E, Varon D and Siegal T: High incidence of thromboembolism in patients with central nervous system lymphoma. Cancer 98: 1239-1242, 2003.

12. Caruso V, Di Castelnuovo A, Meschengieser S, Lazzari MA, de Gaetano G, Storti S, Iacoviello L and Donati MB: Thrombotic complications in adult patients with lymphoma: A meta-analysis of 29 independent cohorts including 18018 patients and 1149 events. Blood 115: 5322-5328, 2010.

13. Ikeda M, Kan-No H, Hayashi M, Tsukada H, Shida M, Hirasawa T, Muramatsu T, Ogushi Y and Mikami M: Predicting perioperative venous thromboembolism in Japanese gynecological patients. PLoS One 9: e89206, 2014.

14. Boersma RS, Hamulyak K, Cate HT and Schouten HC: Congenital thrombophilia and central venous catheter-related thrombosis in patients with cancer. Clin Appl Thromb/Hemost 16: 643-649, 2010.

15. Achkar A, Horellou MH, Leclercq X, Lambert Y, Conard J, Laaban JP and Samama MM: PO73 prevalence of cancer and congenital thrombophilia in 435 patients with acute venous thromboembolism (VTE). Thromb Res 120 (Suppl 2): S168, 2007.

16. Liu BH, Yu H, Tu K, Li C, Li YX and Li YY: DCGL: An R package for identifying differentially coexpressed genes and links from gene expression microarray data. Bioinformatics 26 $2637-2638,2010$

17. Khorana AA, Kuderer NM, Culakova E, Lyman GH and Francis CW: Development and validation of a predictive model for chemotherapy-associated thrombosis. Blood 111: 4902-4907, 2008.

18. Korte W: Cancer and thrombosis: An increasingly important association. Support Care Cancer 16: 223-228, 2008.

19. Rodriguez AO, Wun T, Chew H, Zhou H, Harvey D and White RH: Venous thromboembolism in ovarian cancer. Gyneco Oncol 105: 784-790, 2007.

20. Semrad TJ, O'Donnell R, Wun T, Chew H, Harvey D, Zhou H and White RH: Epidemiology of venous thromboembolism in 9489 patients with malignant glioma. J Neurosur 106: 601-608, 2007.

21. Franchini M: Thromboembolic risk in hematological malignancies. Clin Chem Lab Med 53: 1139-1147, 2015.

22. Falanga A, Marchetti M and Russo L: Venous thromboembolism in the hematologic malignancies. Curr Opin Oncol 24: 702-710, 2012.

23. Elice F and Rodeghiero F: Hematologic malignancies and thrombosis. Thromb Res 129: 360-366, 2012.

24. Kunisawa J and Kiyono H: Alcaligenes is commensal bacteria habituating in the gut-associated lymphoid tissue for the regulation of intestinal IgA responses. Front Immunol 3: 65, 2012.

25. Avula LR, Knapen D, Buckinx R, Vergauwen L, Adriaensen D, Van Nassauw L and Timmermans JP: Whole-genome microarray analysis and functional characterization reveal distinct gene expression profiles and patterns in two mouse models of ileal inflammation. BMC Genomics 13: 377, 2012.
26. Wang $\mathrm{L}$ and $\mathrm{Wu} \mathrm{X}$ : Cytokines of defender against cell death 1 and allograft inflammatory factor-1 with regard to innate immunity of fish. J Fisher Sci Chin 18: 237-242, 2013.

27. Ghasemi S, Tavakoli A, Moghadam M, Zargar MA, Abbaspour M, Hatamnejadian N and Ebrahimi A: Risk of prostate cancer and thrombosis-related factor polymorphisms. Biomed Rep 2: 53-56, 2014

28. Sarajlić A, Janjić V, Stojković N, Radak D and Pržulj N: Network topology reveals key cardiovascular disease genes. PLoS One 8 : e71537, 2013.

29. von Mering C, Krause R, Snel B, Cornell M, Oliver SG, Fields S and Bork P: Comparative assessment of large-scale data sets of protein-protein interactions. Nature 417: 399-403, 2002.

30. Gaiteri C, Ding Y, French B, Tseng GC and Sibille E: Beyond modules and hubs: The potential of gene coexpression networks for investigating molecular mechanisms of complex brain disorders. Genes Brain Behav 13: 13-24, 2014.

31. Sharan R, Ulitsky I and Shamir R: Network-based prediction of protein function. Mol Syst Biol 3: 88, 2007.

32. Yook SH, Oltvai ZN and Barabási AL: Functional and topological characterization of protein interaction networks. Proteomics 4 928-942, 2004

33. Horvath S and Dong J: Geometric interpretation of gene coexpression network analysis. PLoS Comput Biol 4: e1000117, 2008

34. Safari-Alighiarloo N, Taghizadeh M, Rezaei-Tavirani M, Goliaei B and Peyvandi AA: Protein-protein interaction networks (PPI) and complex diseases. Gastroenterol Hepatol Bed Bench 7: 17-31, 2014

35. Jun JI and Lau LF: Taking aim at the extracellular matrix: CCN proteins as emerging therapeutic targets. Nat Rev Drug Discov 10: 945-963, 2011.

36. Hall-Glenn F and Lyons KM: Roles for CCN2 in normal physiological processes. Cell Mol Life Sci 68: 3209-3217, 2011.

37. de Winter P, Leoni P and Abraham D: Connective tissue growth factor: Structure-function relationships of a mosaic, multifunctional protein. Growth Factors 26: 80-91, 2008

38. Yin D, Chen W, O'Kelly J, Lu D, Ham M, Doan NB, Xie D, Wang C, Vadgama J, Said JW, et al: Connective tissue growth factor associated with oncogenic activities and drug resistance in glioblastoma multiforme. Int J Cancer 127: 2257-2267, 2010.

39. Leask A and Abraham DJ: All in the CCN family: Essential matricellular signaling modulators emerge from the bunker. J Cell Sci 119: 4803-4810, 2006.

40. Brigstock DR: Connective tissue growth factor (CCN2, CTGF) and organ fibrosis: Lessons from transgenic animals. J Cell Commun Signal 4: 1-4, 2010.

41. Koshman YE, Patel N, Chu M, Iyengar R, Kim T, Ersahin C, Lewis W, Heroux A and Samarel AM: Regulation of connective tissue growth factor gene expression and fibrosis in human heart failure. J Card Fail 19: 283-294, 2013.

42. Mori T, Kawara S, Shinozaki M, Hayashi N, Kakinuma T, Igarashi A, Takigawa M, Nakanishi T and Takehara K: Role and interaction of connective tissue growth factor with transforming growth factor-beta in persistent fibrosis: A mouse fibrosis model. J Cell Physiol 181: 153-159, 1999.

43. Charrier A and Brigstock DR: Regulation of pancreatic function by connective tissue growth factor (CTGF, CCN2). Cytokine Growth Factor Rev 24: 59-68, 2013.

44. Aikawa T, Gunn J, Spong SM, Klaus SJ and Korc M: Connective tissue growth factor-specific antibody attenuates tumor growth, metastasis, and angiogenesis in an orthotopic mouse model of pancreatic cancer. Mol Cancer Ther 5: 1108-1116, 2006.

45. Sala-Torra O, Gundacker HM, Stirewalt DL, Ladne PA, Pogosova-Agadjanyan EL, Slovak ML, Willman CL, Heimfeld S, Boldt DH and Radich JP: Connective tissue growth factor (CTGF) expression and outcome in adult patients with acute lymphoblastic leukemia. Blood 109: 3080-3083, 2007.

46. Lee AY and Levine MN: Venous thromboembolism and cancer: Risks and outcomes. Circulation 107 (23 Suppl 1): I17-I21, 2003. 